\title{
Gallstone abscess: some drops may end up costlier!
}

\section{Vasanthakumar Venugopal • Sunil Kumar Puri • Abhay Kumar Kapoor • Lalendra Upreti}

Published online: 2 August 2012

(C) Indian Society of Gastroenterology 2012

This 47-year-old female patient presented to the Gastrointestinal Surgery OPD at our hospital with long standing abdominal pain and 2 weeks history of fever. She had undergone laparoscopic cholecystectomy 18 months back. CT scan of the abdomen revealed a $4 \mathrm{~cm} \times 4 \mathrm{~cm}$ well defined extraperitoneal collection located between the left lobe of liver and the anterior abdominal wall compressing the left lobe (Fig. 1). MRI abdomen showed multiple signal void foci in all sequences within the collection (Fig. 2) suggesting the diagnosis of abscess complicating dropped gallstones. Surgical drainage of the abscess with stone retrieval was performed. Intraoperative perforation of the gallbladder and spillage of gallstones is a well-known complication of laparoscopic cholecystectomy, but subsequent abscess formation is unusual [1]. The incidence of

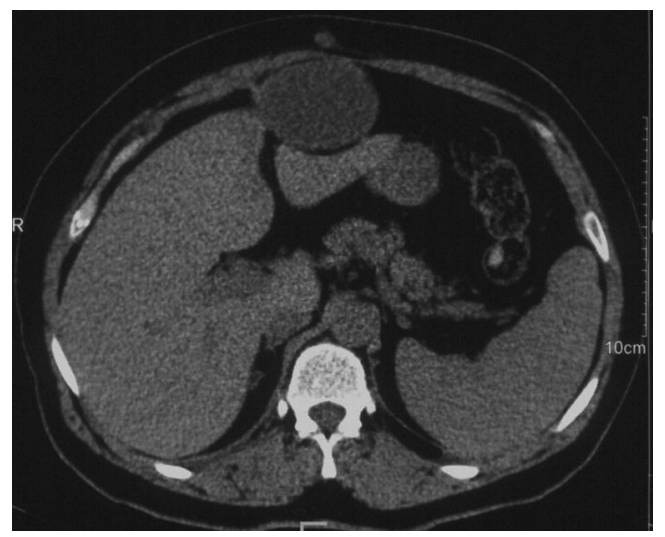

Fig. 1 CT scan showing extraperitoneal collection anterior to liver

V. Venugopal $(\triangle) \cdot$ S. K. Puri $\cdot$ A. K. Kapoor $\cdot$ L. Upreti Department of Radiology, G B Pant Hospital,

Jawahar Lal Nehru Marg,

New Delhi 110 002, India

e-mail: vasanthdrv@gmail.com gallbladder perforation has been estimated to occur during $15 \%$ to $30 \%$ of laparoscopic cholecystectomy. The incidence of intraabdominal abscesses is reported to be $0.6 \%$ in patients who had bile spillage and $2.9 \%$ in those who had both bile and gallstone spillage [2]. Dropped stones are a potential source of recurrent intraabdominal and intrathoracic abscesses. Hence in addition to drainage of abscess, attempts should be made to remove the calculi either percutaneously or by surgery.



Fig. 2 Fat sat T2 weighted MRI showing signal void foci within the collection consistent with calculi

\section{References}

1. MacFadyen BV Jr, Vecchio R, Ricardo AE, Mathis CR. Bile duct injury after laparoscopic cholecystectomy: the United States experience. Surg Endosc. 1998;12:315-21.

2. Rice DC, Memon MA, Jamison RL, et al. Long term consequences of intraoperative spillage of bile and gallstones during laparoscopic cholecystectomy. J Gastrointest Surg. 1997;1:85-91. 